\title{
Beta Anomaly, Mispricing, and Idiosyncratic Volatility in Indonesia
}

\author{
Feliciana Florencia ${ }^{1}$ and Sung Suk Kim ${ }^{2}$ \\ \{felicianaflo@gmail.com ${ }^{1}$, sungsuk.kim@uph.edu ${ }^{2}$ \} \\ Department of Management, Faculty of Economy and Business, \\ Universitas Pelita Harapan, Indonesia ${ }^{1,2}$
}

\begin{abstract}
The purpose of this research is to find the evidence of beta anomaly in Indonesia, a stock return anomaly which happens when the high-beta stocks generate lower alpha than the low-beta stocks, and provide an explanation behind it, during the year 2007-2016. This research use double-sorted portfolio formations to find the presence of beta anomaly, the relationship between stocks' mispricing level and beta, the relationship between stocks' beta and beta anomaly, and the relationship between idiosyncratic volatility (IVOL) and beta anomaly. The findings show the presence of beta anomaly and is caused by IVOL rather than beta, proved by the alpha and IVOL negative relationship across almost all beta quintiles and the disappearance of the anomaly when one controls for IVOL. This anomaly is present in IDX during the period and is significant only at overpriced stocks.
\end{abstract}

Keywords: Beta Anomaly, Mispricing, Idiosyncratic Volatility

1. Introduction

High risk, high return is a long-standing and essential principle of financial economics formalized for the first time by Harry Markowitz in his portfolio theory. Later on, many modern asset pricing theories were born in search of empirical evidence of this risk and return trade-off. One of the first modern asset pricing theory that was developed from this theory and even won the Nobel Prize is the Capital Asset Pricing Model (CAPM). The classic CAPM denotes that expected excess return on any asset is directly proportional to its systematic risk or beta $(\beta)$.

However, various empirical evidence suggests that even though the security market line - the line graph that shows the relationship between beta and the expected return, is generally positive, it is actually flatter than what the model had suggested, or in other words, assets with higher risk often fails to generate higher expected returns, compared to low risk assets. Started by the studies of Black et al. [1], Fama and MacBeth [2], and Haugen and Heins [3], the evidence shows that high-beta stocks poorly performed in comparison to their high beta, while the low-beta stocks actually have higher excess return than the supposedly low return based on their low beta. In other words, beta and alpha have negative relationship - the beta anomaly.

Beside from examining the empirical relation between risk and return in Indonesian stock market, in this paper, author wants to study the role of idiosyncratic volatility (hereinafter referred to as IVOL) behind the anomaly. Following Liu, Stambaugh, and Yuan [4] study who provide the evidence that questioned several empirical evidences focusing on beta as the main culprit of this anomaly beforehand, that beta is not the main reason of beta anomaly, rather it was guilty by association, because beta and IVOL has a positive relationship. They find the evidence that beta anomaly involves mispricing, because the anomaly is only significant within overpriced stocks (Liu et al.)[4]

To the existing literatures, this paper will contribute in two ways, which is, to the 
best of our knowledge, documented the presence of beta anomaly exclusively in Indonesian stock market. This new evidence will benefit academician to further explore this phenomenon in Indonesia and the specific reasons behind this anomaly. Moreover, it can help investors to develop new equity investing strategies in beating the Indonesian stock market and so that they won't follow the high risk-high return strategy blindly. The second one is, this paper will show that, consistent with Liu, et al.[4] empirical evidence on the NYSE/Amex/NASDAQ common stocks, that beta is not the component responsible for beta anomaly, but it is guilty from positive correlation with IVOL.

\section{Empirical Measures: mispricing, beta, IVOL, and alpha}

The population of our research consisted of all listed companies in IDX during the period of ten year (2007-2016), we will make adjustment every year regarding the additional IPOs and delisted companies from IDX as well as excluding penny stocks, stocks with more than two mispricing variables that are unavailable, and stocks with at least 36 months consecutive returns needed to calculate rolling beta, resulting in an unbalanced set of data. After sorting out the sample based on our criteria, we are left with 3193 stocks for ten years, approximately 69 percent of the population.

We follow Stambaugh, Yu, and Yuan [5] in constructing the mispricing measure for each stock, using different anomalies that challenge risk-based model. Stambaugh et al. (2015) [5] There are eleven anomalies used to construct the mispricing measure by Stambaugh, Yu, and Yuan [5] that act as proxies for stocks' ex ante potential to be mispriced. However, because of time and data resources limitation of the author, the four out of eleven anomalies are left out in this study because they are too intricate and time consuming to calculate and need a specific data that is not available in author's data source. They are financial distress by Campbell et al. [6], O-Score bankruptcy probability by Ohlson [7], composite equity issues by Daniel and Titman [8] and momentum effect by Jegadeesh and Titman [9]. We need to calculate each anomaly separately for each stock every year, then we rank them into three categories. We divide them into three categories instead of five like in the original paper because of our number of sample is way smaller than in the U.S stock markets.

We calculate beta following Liu, Stambaugh, and Yuan [4] approach by regressing the stock's monthly excess return on monthly market excess returns. We use six-month Indonesia Government Bond yield as opposed to one-month U.S. T-bill rate. We use a rolling five-year window since in this paper, we want to compare high-beta stock and low-beta stocks. To get reliable estimates on this matter, the Liu, Stambaugh, and Yuan (2018)[4] method is claimed as the best way to calculate the beta. For this method, we need stocks with at least 36 months of returns.

Following Ang et al. [10], we measure a stock's idiosyncratic volatility (IVOL) as the residuals squared from regressing the stock's monthly excess returns on the FamaFrench [11] three factors. We use monthly as opposed to daily because of the data limitation of Fama-French variables which is daily HMB and SML in Indonesia. To calculate Alpha $(\alpha)$ is we regress the excess monthly return of portfolio in a given month with monthly Fama-French three factors. We regress by portfolio to get the constant or intercept as the portfolio alpha for each portfolio for ten years. 


\section{Empirical Results}

This section presents the empirical results. We want to see the difference in the portfolios' alpha and the relation with mispricing using double-sorting method. We also sort on IVOL to see the relation between alpha and IVOL.

\section{a. Beta and Mispricing}

At the end of each month for ten years, we independently sort the stocks based on their pre-ranking betas from the highest to the lowest, forming quintiles. And then independently sort the stocks by their mispricing levels, forming three rankings from the highest to lowest, in respect to their average mispricing score annually based on seven mispricing measures. Next, we form fifteen portfolios consisting the intersection of these two variables and another five sorted by the beta quintiles only. We construct two panels in Table 1, the Panel A contains the total average number of stocks in each doublesorted portfolio, and Panel B consist of the portfolios' beta estimated using simple CAPM regression over 2007-2016 period.

We can see in the Panel A that the higher the beta level, the more unbalanced the distribution of the number of stocks is. And opposite to Liu, Stambaugh, and Yuan (2018) [4] findings in the US stock markets, high-beta stocks (quintile one) are the most prevalent among the underpriced stocks in Indonesia rather than among the overpriced stocks (19 versus 12). This tells us that in Indonesia, the high-beta stocks are not exclusively over- priced, but rather underpriced. Panel B also suggest the estimated beta for the highest beta quintiles is biggest among stocks in the second mispricing level, compared to over- priced stocks (1.97 versus 1.83). Again, telling us that in Indonesia, different with the US stock markets, the overpriced stocks have the lowest beta. This is possible because they are two different stock markets and have different characteristics. Another information that we can gather from this table is that in the last row, the difference in the estimated beta of all stocks between the highest and lowest quintiles is very big (2.15), unlike the U.S stock market which has lesser spread of 0.92. This phenomenon in Indonesian stock market occurs mainly because of the huge presence of illiquid stocks and unusual market activities.

\section{b. Beta Anomaly and Mispricing}

In Table 2, we have the portfolios' alpha computed with Fama-French three factor model regression and the $t$-statistics in parentheses. In the last row, we can see the alphas across different level of beta, we can clearly see that stocks in the highest beta quintiles underperformed the lowest beta quintiles by -1.4 bps (0.012 versus 0.026$)$ with a $t$-statistics of -2.13 . These numbers show that beta anomaly exists within the overall stocks at IDX, and both economically and statistically significant. Conforming with Liu, Stambaugh, and Yuan (2018) and numerous previous evidences across stock markets, be it developed or emerging markets, we accept the first hypothesis one that beta anomaly is present in Indonesian stock market. 
TABLE 1

Average Number of Stocks and Estimated Beta of the Double-sorted Portfolio based on Mispricing Levels and Pre-ranking Betas

\begin{tabular}{|c|c|c|c|c|c|c|}
\hline \multirow{2}{*}{ Mispricing Level } & \multicolumn{5}{|c|}{ Beta quintiles } & \multirow{2}{*}{ Highest - Lowest } \\
\hline & Highest & 2 & 3 & 4 & Lowest & \\
\hline \multicolumn{7}{|c|}{ Panel A: Average Number of Stock } \\
\hline Overpriced & 12 & 13 & 13 & 16 & 17 & \\
\hline 2 & 18 & 16 & 16 & 15 & 17 & \\
\hline Underpriced & 19 & 20 & 21 & 19 & 17 & \\
\hline \multicolumn{7}{|c|}{ Panel B : Estimated Beta } \\
\hline Overpriced & 1.83 & 1.23 & 0.79 & 0.45 & -0.39 & 2.22 \\
\hline 2 & 1.97 & 1.31 & 0.85 & 0.48 & -0.11 & 2.08 \\
\hline Underpriced & 1.88 & 1.41 & 1.02 & 0.49 & -0.52 & 2.41 \\
\hline All stocks & 1.87 & 1.33 & 0.91 & 0.46 & -0.28 & 2.15 \\
\hline
\end{tabular}


TABLE 2

Alphas of the Double-sorted Portfolios based on Mispricing Levels and Pre-ranking Betas

\begin{tabular}{lcccccc}
\hline \multicolumn{7}{c}{ Beta quintiles } \\
Mispricing Level & Highest & 2 & 3 & 4 & Lowest & Highest-Lowest \\
Overpriced & -0.003 & 0.004 & 0.011 & 0.004 & 0.018 & -0.021 \\
& $(-0.52)$ & $(0.77)$ & $(1.64)$ & $(1.03)$ & $(2.79)$ & $(-2.30)$ \\
2 & 0.018 & 0.007 & 0.012 & 0.012 & 0.026 & -0.008 \\
& $(2.99)$ & $(1.54$ & $(2.90)$ & $(2.55)$ & $(5.77)$ & $(-1.03)$ \\
Underpriced & 0.021 & 0.014 & 0.018 & 0.014 & 0.039 & -0.018 \\
& $(3.38)$ & $(3.25)$ & $(4.72)$ & $(3.46)$ & $(5.04)$ & $(-1.78)$ \\
All stocks & 0.012 & 0.009 & 0.014 & 0.010 & 0.026 & -0.014 \\
& $(2.52)$ & $(2.30)$ & $(4.24)$ & $(3.73)$ & $(5.57)$ & $(-2.13)$ \\
\hline
\end{tabular}

c. Evidence of IVOL's Role on Beta Anomaly

Our hypothesis follows Liu, Stambaugh, and Yuan (2018)[4] theory that says beta is not the component responsible for beta anomaly to occur but IVOL is. In order to prove that, we construct Table 3 which reports the alpha of our new portfolios, formed by deleting the overpriced high-IVOL stocks, which means that every stock that's inside the first mispricing level and first IVOL ranking is deleted, to see the IVOL effect on beta anomaly in all stocks. If the anomaly becomes stronger, it means that IVOL is not the one that causing beta anomaly, but if the beta anomaly becomes missing, then the IVOL effect is the cause of beta anomaly. We calculated IVOL in respect to Fama French three factor model and square the residuals for each stock, then we divide them into three categories.

TABLE 3

Alphas of the Double-sorted Portfolios based on Mispricing Levels and Pre-ranking Betas after Deleting Overpriced High-IVOL Stocks

\begin{tabular}{lrccccc}
\hline \multirow{2}{*}{ Mispricing Level } & Highest & 2 & 3 & 4 & Lowest & Highest - Lowest \\
Overpriced & 0.007 & 0.017 & 0.017 & 0.016 & 0.016 & -0.010 \\
& $(1.95)$ & $(4.62)$ & $(4.22)$ & $(3.84)$ & $(3.57)$ & $(-1.66)$ \\
2 & 0.018 & 0.007 & 0.012 & 0.012 & 0.026 & -0.008 \\
\multirow{3}{*}{ Underpriced } & $(2.99)$ & $(1.54)$ & $(2.90)$ & $(2.55)$ & $(5.77)$ & $(-1.03)$ \\
& 0.021 & 0.014 & 0.018 & 0.014 & 0.039 & -0.018 \\
\multirow{2}{*}{ All stocks } & $(3.38)$ & $(3.25)$ & $(4.72)$ & $(3.46)$ & $(5.04)$ & $(-1.78)$ \\
& 0.0154 & 0.011 & 0.015 & 0.012 & 0.025 & -0.009 \\
& $(3.56)$ & $(3.11)$ & $(4.91)$ & $(4.27)$ & $(5.72)$ & $(-1.52)$ \\
\hline
\end{tabular}

In this table, we can see in the last row that the high-low alpha spread is more positive $(-0.009)$ than Table $2(-0.014)$, and it is not statistically significant, meaning that there is no beta anomaly if we remove the overpriced, high-IVOL stocks. Therefore, we can conclude that there is IVOL effect on beta anomaly, because if we exclude overpriced high-IVOL stocks, the beta anomaly become non-existent. Which is consistent with Liu, Stambaugh, and Yuan (2018) findings in the U.S stock markets that the real reason behind beta anomaly is IVOL, not beta. However, beta is seen as the reason because of the positive relationship between beta and IVOL. 
TABLE 4

Alphas of the Double-sorted Portfolios based on Mispricing Levels and Pre-ranking Betas after Deleting High-beta Stocks

\begin{tabular}{lcccccc}
\hline \multirow{2}{*}{ Mispricing Level } & Highest & 2 & 3 & 4 & Lowest & Highest-Lowest \\
Overpriced & 0.006 & 0.010 & 0.000 & 0.001 & 0.026 & -0.020 \\
& $(1.08)$ & $(1.64)$ & $(-0.02)$ & $(0.20)$ & $(3.40)$ & $(-2.03)$ \\
2 & 0.006 & 0.010 & 0.016 & 0.010 & 0.027 & -0.022 \\
\multirow{3}{*}{ Underpriced } & $(1.08)$ & $(2.11)$ & $(3.41)$ & $(2.07)$ & $(5.58)$ & $(-3.11)$ \\
& 0.013 & 0.017 & 0.012 & 0.021 & 0.047 & -0.035 \\
\multirow{2}{*}{ All stocks } & $(3.19)$ & $(3.91)$ & $(3.32)$ & $(3.46)$ & $(5.15)$ & $(-3.43)$ \\
& 0.0089 & 0.012 & 0.010 & 0.009 & 0.031 & -0.022 \\
& $(2.17)$ & $(3.68)$ & $(3.52)$ & $(2.90)$ & $(6.15)$ & $(-3.40)$ \\
\hline
\end{tabular}

To provide further evidence of beta insignificance to the beta anomaly, we construct new portfolios after eliminating the stocks on the highest beta quintile as shown in Table 4. If beta is indeed the one who responsible of beta anomaly, the absence of high-beta stocks should make the anomaly less significant. But unlike the result in Table 3, the beta anomaly is in fact stronger than ever, with the overall stocks' alpha difference of -0.022 between the highest beta and lowest beta quintiles and $t$-statistic of -3.40 . This proves that the anomaly is getting stronger and more significant without the presence of highbeta stocks, supporting our first evidence in Table 3 that IVOL is indeed the real reason behind beta anomaly, not beta itself. This evidence is consistent with Liu, Stambaugh, and Yuan (2018) finding in the U.S stock market that beta anomaly is still strong despite the range of beta is reduced. These two evidences lead us to accept our third hypothesis.

\section{d. Further Evidence of IVOL's Role on Beta Anomaly}

For beta anomaly to occur, there should be a negative relationship between alpha and IVOL. This relationship and the significance of IVOL role to the beta anomaly can be seen by forming double-sorted portfolio based on IVOL level and pre-ranking beta. In Table 5, we construct 15 portfolios of the intersection of IVOL and pre-ranking betas, the spreads, and the average alpha of each level of variables.

The negative relationship of alpha and IVOL can be observed from the second to last row, where the high-low alpha spread is negative across almost beta quintiles, statistically significant in the second beta quintile $(-2.86)$. Unlike the high-low alpha spread across IVOL level, where all of them are negative but less significant, showing less significant beta anomaly once one controls for IVOL. From this evidence we can conclude that thereis beta anomaly and the relationship between alpha and IVOL is indeed negative, therefore proving our third hypothesis true, that IVOL is the component that responsible for beta anomaly, not beta. This evidence in Indonesia is consistent with the U.S. where Liu, Stambaugh, and Yuan (2018) proves that beta is only guilty by association to the real culprit behind beta anomaly, which is IVOL. 
TABLE 5

Alphas of the Double-sorted Portfolios based on IVOL Levels and Pre-ranking betas

\begin{tabular}{lcllllll}
\hline \multirow{2}{*}{ IVOL Level } & \multicolumn{2}{l}{ Beta quintiles } & & & & \\
& Highest & 2 & 3 & 4 & \multicolumn{2}{l}{ Lowest Highest-Lowest } & Average \\
High & 0.021 & 0.014 & 0.037 & 0.036 & 0.070 & -0.05 & 0.04 \\
& $(1.63)$ & $(1.48)$ & $(3.31)$ & $(3.66)$ & $(5.54)$ & $(-2.73)$ & $(6.41)$ \\
2 & 0.003 & 0.009 & 0.010 & 0.011 & 0.02 & -0.01 & 0.01 \\
& $(0.59)$ & $(1.84)$ & $(2.26)$ & $(2.46)$ & $(3.34)$ & $(-1.95)$ & $(4.39)$ \\
Low & 0.039 & 0.042 & 0.043 & 0.045 & 0.046 & -0.01 & 0.04 \\
& $(21.24)$ & $(30.49)$ & $(27.90)$ & $(19.90)$ & $(10.80)$ & $(-1.52)$ & $(36.96)$ \\
Highest - Lowest & -0.018 & -0.028 & -0.007 & -0.009 & 0.024 & -0.042 & \\
& $(-1.38)$ & $(-2.86)$ & $(-0.58)$ & $(-0.91)$ & $(1.81)$ & $(-2.27)$ & \\
Average & 0.019 & 0.018 & 0.026 & 0.025 & 0.040 & & \\
& $(4.59)$ & $(5.27)$ & $(6.66)$ & $(6.41)$ & $(7.69)$ & & \\
& & & & & & &
\end{tabular}

4. Conclusions

This research is done to find the presence of beta anomaly in Indonesia, a stock return anomaly which happens when the high-beta stocks generate lower alpha than the low-beta stocks, and provide an explanation behind it, during the year 2007-2016. This research use double-sorted portfolio formations to find the presence of beta anomaly, the relationship between stocks' mispricing level and beta, the relationship between stocks' beta and beta anomaly, and the relationship between IVOL and beta anomaly.

Based on the evidence shown on the result, this research concludes that high-beta stocks are the most prevalent among the underpriced stocks, opposite to the U.S. evidence, revealing that in Indonesia, underpriced stocks have higher beta than overpriced stocks. Another evidence also shows the huge difference between the highest and lowest portfolio beta is quite big, unlike in the U.S. This phenomenon in Indonesia mainly due to the huge presence of illiquid stocks and unusual market activities.

We find also that there is a presence of beta anomaly in Indonesia within the overall stocks at IDX. Moreover, the evidence shows the alpha difference between the highest and lowest beta quintiles, exists only within the most overpriced stocks. This evidence supports beta anomaly is only significant among the most overpriced stocks as US stock market. This means that in Indonesia, high arbitrage risk or high IVOL which are positively correlated with beta, also deters arbitrageurs to do the transaction like in the U.S. that causing mispricing and there are arbitrage asymmetry effect thus creating a stronger negative alpha and IVOL relationship (beta anomaly) in overpriced stocks.

This research also proves that IVOL is the component responsible for beta anomaly to occur because without the presence of overpriced high-IVOL stocks, the beta anomaly disappeared, and vice versa, without the presence of high-beta stocks, the anomaly become stronger economically and statistically, showing no beta effect on the anomaly. This evidence means that hypothesis three is accepted, that there is IVOL effect in beta anomaly and the real reason behind beta anomaly is IVOL, not beta. However, beta is seen as the reason because beta is positively correlated with IVOL. 


\section{References}

[1] Black, F., Jensen, M.C., and Scholes, M.S. The capital asset pricing model: some empirical tests, in Studies in the Theory of Capital Markets. Praeger (ed. J.M. C), New York, pp. 79-121. (1972)

[2] Fama, E.F. and MacBeth, J.D. Risk, return, and equilibrium: Empirical tests. Jour- nal of political, 81 (3), 607-636. (1973)

[3] Haugen, R.A. and Heins, A.J. Risk and the rate of return on financial assets: Some old wine in new bottles. Journal of Financial and Quantitative Analysis, 10 (5), 775- 784. (1975)

[4] Liu, J., Stambaugh, R.F., and Yuan, Y. Absolving beta of volatility's effects. Jour- nal of Financial Economics, 128 (1), 1-15. (2018)

[5] Stambaugh, R.F., Yu, J., and Yuan, Y. Arbitrage asymmetry and the idiosyncratic volatility puzzle. The Journal of Finance, 70 (5), 1903-1948. (2015)

[6] Campbell, J.Y., Hilscher, J., and Szilagyi, J. In search of distress risk. The Journal of Finance, 63 (6), 2899-2939. (2008)

[7] Ohlson, J.A. (1980) Financial ratios and the probabilistic prediction of bankruptcy. Jour- nal of accounting research, pp. 109-131.

[8] Daniel, K. and Titman, S. Market reactions to tangible and intangible information. The Journal of Finance, 61 (4), 1605-1643. (2006)

[9] Jegadeesh, N. and Titman, S. Returns to buying winners and selling losers: Implications for stock market efficiency. The Journal of finance, 48 (1), 65-91. (1993)

[10] Ang, A., Hodrick, R.J., Xing, Y., and Zhang, X. The cross-section of volatility and expected returns. The Journal of Finance, 61 (1), 259-299. (2006)

[11] Fama EF, French KR. Common risk factors in the returns on stocks and bonds. Journal of financial economics. 1;33(1):3-56. (1993) 